\title{
Gerald Nepom puts the FOCIS on immunology
}

$T_{\text {h }}$

he Federation of Clinical Immunology Societies (FOCIS), an umbrella organization of 40-plus professional societies recently held its annual meeting in San Francisco. The JCI had the opportunity to speak with the current president, Gerald Nepom (Figure 1), who also acts as the director of the Benaroya Research Institute in Seattle.

JCI: Tell us a little about FOCIS.

Nepom: FOCIS is a federation with over 40 professional societies that represent the major clinical and research specialties invested in translational immunology research. FOCIS provides a set of core resources and programs that link together the immunological interests across a wide range of areas of medical subspecialization. The annual FOCIS scientific meeting is built around interdisciplinary themes, such as immunotherapy, immunodiagnosis, host defense, and immunoregulation, rather than in the traditional medical specialty areas. In addition to the annual meeting, FOCIS conducts several very popular courses - one to teach physicians the fundamentals of immunology, one to teach research fellows about translational and clinical immunology, and our newest course, "Interventional Immunology" to keep the community abreast of the rapidly changing use of novel therapeutics directed at immune pathways. Another major

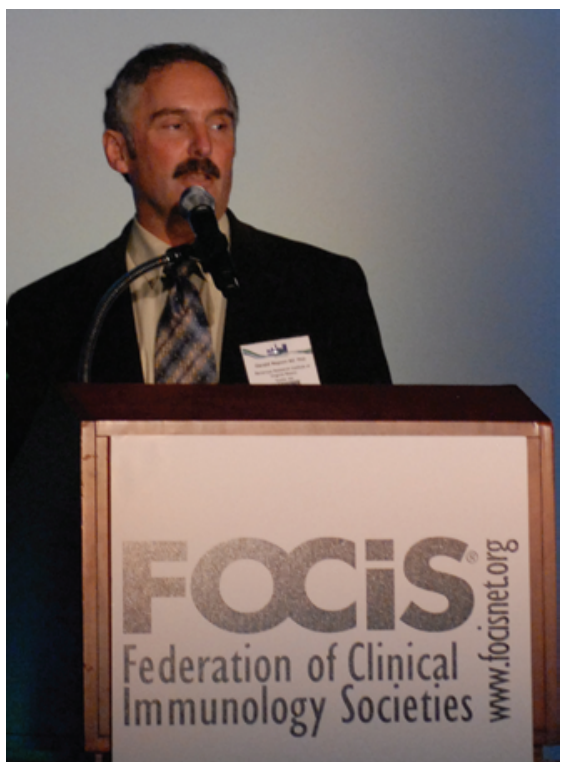

Figure 1

Gerald Nepom at the 2009 FOCIS meeting.
FOCIS program is our network of academic immunology centers, called FOCIS Centers of Excellence, which utilize training opportunities for fellows and exchange research and teaching materials.

JCI: How are the Centers of Excellence determined?

Nepom: There are now 57 FOCIS Centers of Excellence, 38 in the United States and 19 in Europe. An application process (http://www.focisnet.org) delineates a very specific set of requirements for this designation, including the ability to conduct clinical trials in at least 3 diverse immunological disease areas, a commitment to conduct interdisciplinary translational immunology seminars or grand rounds programs, and so forth.

JCI: Who designs and carries out the trainee initiatives?

Nepom: The FOCIS educational program is very popular, clearly meeting a major unmet need in our trainee constituencies. It is run by a FOCIS committee led by Abul Abbas and Andrew Lichtman.

JCI: As president this year, you had the enormous task of organizing the annual meeting. What issues did you encounter in that process?

Nepom: The biggest challenge for the annual meeting is to anticipate several months in advance what areas of clinical and translational science are likely to be truly exciting and important across disciplines, deserving attention during the meeting. We use an outreach approach through our program committee and member societies, which was chaired by Jacques Banchereau, to guide this activity.

JCI: Given that FOCIS is geared toward clinical immunology, many of the speakers also presented data from animal models - did you encourage speakers to focus on both basic and clinical presentations?

Nepom: The FOCIS perspective is to focus on translational concepts with interdisciplinary clinical relevance, but often mechanistic studies are grounded in preclinical models or basic science. Therefore, we do indeed want the basic work to be presented as a backdrop, but it is only the beginning.

JCI: I note that many of the talks related to genetics, genomics, and genome-wide association studies. Was this done on purpose?

Nepom: Yes. Over the last 3 years, FOCIS played a major role in bringing together investigators from diverse genome-wide association studies in immune-mediated and autoimmune diseases, through a program called the FOCIS Network of Consortia (NOC). This has included data sharing and presentations at the annual meeting to assist the large international genomics consortia in multiple sclerosis, diabetes, lupus, arthritis, Crohn's disease, and others; to exchange methods and results; and speed the dissemination of results. At this point, the effort is largely complete, as most genes with significant odds ratios greater than 1.3 have been identified for the major autoimmune diseases, so this was highlighted at the meeting.

JCI: So what will FOCIS be focusing on next?

Nepom: There is a lot of interest in sharing information regarding best practices for human immune monitoring, particularly exploiting technologies such as multiparameter flow cytometry and arrays. There may also be some opportunities to intersect this type of work with the NOC genotype studies to accelerate the field's interests in genotypeto-phenotype translational research.

JCI: What were the scientific highlights for you? Any presentations you found most compelling?

Nepom: I particularly enjoy presentations that highlight a research insight that led to a successful therapeutic approach. Examples include Jeff Ravetch's (The Rockefeller University) work on Ig glycans and mechanism of IVIG therapy; also Steve Holland's (NIAID) work drawing from human experiments of nature - rare mutations in genes of immunologic interest - to identify targets for therapeutics. I also enjoy presentations that highlight a conundrum - sometimes where mouse and man are very different - because this helps guide future work in productive directions.

JCI: Will you be happy to hand over the presidential reins?

Nepom: I've been FOCIS president for 2 years and feel privileged to have helped guide the organization during a very exciting time. There is no shortage of opportunities to contribute to strategic initiatives in our field, and in particular I am looking forward to continuing to build programs at the Benaroya Research Institute.

\section{Ushma S. Neill}

